\title{
EDITORIAL
}

\section{The appeal and challenges of an integrative approach to psychiatry}

\author{
Rafael Ambríz González, Lisa Bortolotti iD \\ University of Birmingham, Birmingham, UK.
}

Problems of Living ${ }^{1}$ is an impressive and ambitious project, in scope and goal. In just over 300 pages, Dan Stein covers the most controversial debates in psychiatry, from a point of view that is neither clinical nor psychological, but philosophical. As Stein emphasizes in his editorial, the goal is to mediate between traditional and critical accounts of psychiatry to offer a more balanced view of the discipline. In pursuing this goal, Stein does not shun from big questions, but faces them head-on, from the relationship between mind and body to how to pursue happiness and meaning in life. The discussion is not merely an overview and quick assessment of the most influential positions in these long-standing debates, but a passionate, in-depth defense of a new, potentially transforming approach that overcomes sterile polarization and is genuinely pluralistic about methodology.

An example of the integrative approach is offered in chapter 7, where Stein identifies two conceptual frameworks "guiding the future of psychiatry" (page 181). ${ }^{1}$ For clinical neuroscience progress will be made by finding well-established biomarkers linked to specific psychiatric conditions - a task that has been the main focus of psychiatry for decades. In contrast, for global mental health, psychiatry will advance by identifying social determinants of psychiatric conditions, and "by developing interventions that are feasible and acceptable around the world" (page 182). Stein proposes that psychiatry will make better progress by adopting an integrative approach. This requires recognizing that psychiatry has a "range of gaps," those identified by clinical neuroscience and those identified by global mental health - and all the gaps need to be filled. The picture Stein sketches is inspiring, leading to a more personalized and comprehensive notion of public health, but his proposal also faces significant challenges.

There are two tensions involved. A first tension emerges in the characterization of the scope of psychiatry, which is instrumental to a detection of the future directions for the discipline. Traditionally, clinical psychiatry deals with mental disorders associated with putative biological dysfunctions. But even some research programs within biological psychiatry call for a substantial reframing of such a scope. For instance, projects in this area, such as the Research Domain Criteria developed by the U.S. National Institute of Mental Health, are not based

Correspondence: Lisa Bortolotti, Philosophy Department, University of Birmingham, Edgbaston, B15 2TT, Birmingham, UK.

E-mail: I.bortolotti@bham.ac.uk

Submitted Jul 30 2021, accepted Jul 30 2021, Epub Oct 292021. on the search for biomarkers for specific mental disorders, as noted elsewhere. ${ }^{2}$ Advocates of global mental health also propose that we reframe our understanding of the scope of psychiatry, emphasizing instead the role of social and environmental aspects. Some warn against underdiagnosis and undertreatment, stretching the notion of health to the point that it almost overlaps with that of agency, and issues such as poverty and thriving become legitimate health issues - as in holistic models such as Nordenfelt's. ${ }^{3}$ Other researchers want to shrink the notion of health to endorse a normalization of human suffering and a de-medicalization of psychiatry - as in the UK British Psychological Society's Power Threat Meaning framework. ${ }^{4}$ Thus, the idea that the scope of psychiatry is determined by mental disorders is a matter of dispute within clinical neuroscience and within competing versions of global mental health. How can the integrative approach provide a coherent way to pursue personalized public mental health when the very boundaries of what counts as health are contested?

A second tension concerns the nature of the various levels of explanation at which psychiatry currently operates and the relative methodological demands. Clinical neuroscience focuses on the biological level - in particular on neurobiochemical and genetic processes - whereas global mental health addresses social and environmental factors. It is plausible that neurobiochemical and genetic processes respond to principles in biochemistry and genetics, whereas social and environmental factors are governed by principles studied by the social and human sciences. Here, the challenge for an integrative approach is to provide a coherent unified methodology that can offer an explanation of the phenomena observed at both levels and account for the relationship between them. ${ }^{5}$ This is a complicated task because, for clinical neuroscience, the biological level appears to be fundamental, but, for global mental health, social and environmental factors are fundamental. The pluralism of the integrative approach tells us that both approaches are right, but will this provide enough guidance as to how to combine the methods of clinical neuroscience and global mental health? How will the ensuing complexity be accommodated in an overall coherent view?

A clue to a possible resolution comes from Stein's enlightened vision of science which transpires through the

How to cite this article: Ambríz González R, Bortolotti L. The appeal and challenges of an integrative approach to psychiatry. Braz J Psychiatry. 2022;44:231-232. http://dx.doi.org/10.1590/1516-44462021-0035 
whole book. Stein consistently, and in our view correctly, challenges the perceived gulf between the physical sciences, with their aura of objectivity and timelessness, and the human and social sciences, with their replication crises and dubious epistemic credentials. Contrary to the stubborn Popperian tradition in the philosophy of science, Stein shows that when science is broadly conceived as practice, as an activity, ${ }^{6}$ at the same time theory-bound and value-laden, it can shed light on both physical and social processes, and help us explain causation and meaning, as long as transparency and fallibility are embraced.

\section{Disclosure}

The authors report no conflicts of interest.

\section{References}

1 Stein D. Problems of living. Rio de Janeiro: Elsevier; 2021.

2 Insel TR, Cuthbert BN. Endophenotypes: bridging genomic complexity and disorder heterogeneity. Biol Psychiatry. 2009;66: 988-9.

3 Nordenfelt L. Towards a holistic theory of health. In: Nordenfelt L, editor. On the nature of health: an action-theoretic approach. Vol 26. Dordrecht: Springer; 1987. p. 35-104.

4 Johnstone L, Boyle M. The power threat meaning framework: an alternative nondiagnostic conceptual system. J Humanist Psychol. 2018. p. 2216781879328.

5 Broome M, Bortolotti L. Psychiatry as cognitive neuroscience: philosophical perspectives. Oxford: Oxford University Press; 2009.

6 Bortolotti L, Heinrichs B. Delimiting the concept of research: an ethical perspective. Theor Med Bioeth. 2007;28:157-79. 